\title{
GENERAL PROPERTIES OF GIANT RADIO GALAXIES
}

\author{
U. KLEIN AND K.-H. MACK \\ Radioastronomisches Institut der Universität Bonn - Auf dem \\ Hügel 71, 53121 Bonn, Germany \\ AND \\ L. SARIPALLI \\ Indian Institute of Astrophysics - Koramangala, Bangalore - \\ 560034, India
}

\section{Introduction}

So far, the number of so-called giant radio galaxies (GRGs) is small. We define them as radio sources with linear sizes larger than $1 \mathrm{Mpc}\left(\mathrm{H}_{0}=\right.$ $75 \mathrm{~km} \mathrm{~s}^{-1} \mathrm{Mpc}^{-1}$ ). On-going low-frequency surveys may come up with many more candidates for this species. Their very existence may ultimately be connected with their environment. For instance, for a source like 3C236, which exhibits a linear and undisturbed structure over some $4 \mathrm{Mpc}$, the surrounding medium must have a low density. GRGs may thus serve to probe large volumes of the most tenuous intergalactic medium (IGM), e.g. via depolarization studies.

Previous investigations of the large-scale properties of GRGs were restricted to frequencies $\leq 1 \mathrm{GHz}$. Owing to their large (angular) sizes, aperture synthesis techniques become quickly insensitive to their large-scale components as one goes to higher frequencies. The need for single-dish work is then obvious.

\section{Effelsberg projects}

Extensive mapping programs have been carried out in the recent past with the Effelsberg $100-\mathrm{m}$ telescope in order to obtain sensitive images in all Stokes parameters of the northern sky GRGs. These high-dynamic range maps complement ideally the low-frequency data obtained with the WSRT (see e.g. Willis and O'Dea, 1990). Maps at 2.7, 4.75 and $10.55 \mathrm{GHz}$ are now available for the GRGs NGC315, NGC6251, DA240, 3C130, 3C236, 3C326, 
4C73.08, 8C0821+695, 0503-286, 1331-099, 1245+67, 4C34.47, 4C 39.04, $4 \mathrm{C} 74.26$, and $1358+305$. For most of these WSRT measurements at $\lambda \lambda 92$ and/or $49 \mathrm{~cm}$ have also been obtained (partly previously published by other authors). These can be used to derive relevant spectral and polarization parameters. Here we report first results on the depolarization characteristics of the lobes of GRGs as derived from the comparison of low- and highfrequency data. Part of our high-frequency mapping programme of GRGs and first results have been published by Klein et al. (1994), Saripalli et al. (1995) and Parma et al. (1996).

\section{First results}

A first glance at the radio maps of GRGs show that they are individuals. They are rarely symmetric, some have complex structures (e.g. NGC6251), some are narrow and straight (e.g. 3C236), some are fat doubles (e.g. DA240). The question arises what makes them look so different, and why they often show asymmetries. If it is the influence of the IGM, our multifrequency measurements should allow us to investigate this. We have therefore begun to analyze their depolarization as a function of wavelength, in order to look for any asymmetries which could be linked to any other asymmetry, viz. morphology, jet sidedness, magnetic field structure or spectral index. The fact that linear polarization is generally rather strong in GRGs even at large wavelengths already implies that the matter density of the surrounding IGM must be relatively low, inferring $\mu \mathrm{G}$ magnetic field strengths. Our preliminary analysis indicates, however, that there is not a clear relation of depolarization asymmetries with any other differences in the lobes of GRGs. The deduced number densities of thermal electrons are in the range between 1 and a few $10^{-5} \mathrm{~cm}^{-3}$. An important conclusion at this stage is that the density of the environment around GRGs is not extraordinarily tenuous as one might expect. Detailed analyses of the maps are underway, and final conclusions may not be drawn before higher resolution polarization studies (e.g. with the VLA), which may eventually resolve any existing Faraday screens, will be made. These studies will also be aided by ongoing evaluations of the ROSAT data base, which aim at detecting hot gas that may surround GRG host galaxies.

\section{References}

Klein U., Mack K.-H., Strom R., Wielebinski R., and Achatz U. (1994) $A \& A, 283,729$

Parma P., de Ruiter H. R., Mack K.-H., van Breugel W., Dey A., Fanti R. and Klein U. (1996), $A \& A$, in press

Saripalli L., Mack K.-H., Klein U., Strom R. and Singal A. K. (1995) $A \mathfrak{E} A$, in press

Willis A. G. and O'Dea C. P. (1990) IAU Symp., 140, 455 